\title{
ARTICLE
}

Epidemiology

\section{Impact of HPV vaccination on cervical screening performance: a population-based cohort study}

\author{
Jiayao Lei $\mathbb{D}^{1}$, Alexander Ploner ${ }^{1}$, Matti Lehtinen ${ }^{2,3}$, Pär Sparén ${ }^{1}$, Joakim Dillner ${ }^{3,4}$ and K. Miriam Elfström ${ }^{3,5}$
}

BACKGROUND: Human papillomavirus (HPV) vaccination is predicted to lower the positive predictive value (PPV) of cytology. METHODS: We included 153,250 girls born between 1989 and 1993, resident in Sweden since the introduction of HPV vaccines (October 2006) and attending cervical screening at age 23 years. We assessed their first cytology and following histopathological diagnosis using Swedish National Cervical Screening Registry (NKCx). By linkage with the national Swedish HPV vaccination registry, we determined PPV of abnormal cytology for cervical intraepithelial neoplasia grade 2 or worse $(\mathrm{CIN} 2+)$ and the differences with 95\% confidence intervals (Cls) according to vaccination status.

RESULTS: The PPV of high-grade cytology for CIN2+ was $69.9 \%(95 \% \mathrm{Cl}, 67.9-71.9), 64.9 \%(95 \% \mathrm{Cl}, 59.8-69.8)$ and $57.4 \%(95 \% \mathrm{Cl}$, 50.9-63.7) among women unvaccinated, initiating vaccination at age 17-22 years and initiating vaccination before age 17 years, corresponding to reduction in PPV by $8 \%(95 \% \mathrm{Cl}, 0-15 \%)$ and $17 \%(95 \% \mathrm{Cl}, 7-26 \%)$ in vaccinated groups after adjustment for birth cohort, respectively.

CONCLUSION: The PPV of cytology for CIN2+ decreased among vaccinated women, and the decrease was stronger for girls vaccinated at younger ages. A switch from cytology to HPV testing might potentially improve the screening performance.

British Journal of Cancer (2020) 123:155-160; https://doi.org/10.1038/s41416-020-0850-6

\section{BACKGROUND}

High-risk human papillomavirus (HPV) is the major cause of cervical cancer. ${ }^{1}$ As of October 2018, 91 countries had introduced HPV vaccines in their national immunisation programmes. ${ }^{2}$ In Sweden, HPV vaccines were introduced in late 2006, and starting from May 2007, HPV vaccination was subsidised for girls aged 13-17 years (birth cohorts 1989-1993). In 2012, a free-of-charge catch-up HPV vaccination programme for girls aged 13-18 years (birth cohorts 1993-1998), and a school-based HPV vaccination programme for girls aged 10-12 years (birth cohorts 1999 onwards) was launched. ${ }^{3}$ The effectiveness of HPV vaccine against genital warts and cervical intraepithelial neoplasia grade 2 or worse $(\mathrm{CIN} 2+)$ observed in Swedish population ${ }^{4-6}$ was in line with a recent meta-analysis, ${ }^{7}$ and the effectiveness increases with younger age at initiating vaccination. Strongest effectiveness has been seen in girls initiating vaccination before age 17 years, with $64 \%$ effectiveness against CIN2+. ${ }^{4,5}$ Vaccination also provides some cross-protection against HPV types not included in the vaccines. $^{8-10}$

According to European Union screening guidelines, women below age 30 years should be screened with cytology ${ }^{11}$ due to the high prevalence of HPV in this age group. In Sweden, cytology screening is recommended for women aged 23-29 years, and primary HPV screening is recommended for women aged 30 years and above. The positive predictive value (PPV) of cytology depends on the prevalence of cervical lesions and has been predicted to decline in vaccinated populations. ${ }^{12}$ As high PPV is essential for achieving a favourable balance between health gains in detecting high-grade cervical lesions and adverse outcomes such as unnecessary referrals, such a decline of PPV could impact screening policies for young women. The aim of our study was to evaluate whether HPV vaccination does indeed affect the PPV of abnormal cytology for CIN2+.

\section{METHODS}

Study population

The identities of all women born between 1 January 1985 and 31 December 1999 were retrieved from the Total Population Register (Fig. 1). ${ }^{13}$ Women who immigrated to Sweden after the introduction of HPV vaccines (1 October 2006), women who emigrated, died or were lost to follow-up before the introduction of HPV vaccines and women who had invalid date of vaccination were excluded, leaving the rest of the birth cohorts eligible for the analysis of HPV vaccination coverage.

For the analysis of detection rates and PPV of cytology for CIN2+, we included women born between 1 January 1989 and 31 December 1993 for whom the vaccination was available through either the subsidised opportunistic programme or the free-ofcharge catch-up programme. In these birth cohorts, we identified women who attended cervical screening at age 23 years through linkage with the Swedish National Cervical Screening Registry

\footnotetext{
${ }^{1}$ Department of Medical Epidemiology and Biostatistics, Karolinska Institutet, SE-171 77 Stockholm, Sweden; ${ }^{2}$ Faculty of Social Sciences, University of Tampere, SE-330 14 Tampere, Finland; ${ }^{3}$ Department of Laboratory Medicine, Karolinska Institutet, SE-141 83 Stockholm, Sweden; ${ }^{4}$ Karolinska University Laboratory, Karolinska University Hospital, SE17176 Stockholm, Sweden and ${ }^{5}$ Regional Cancer Center Stockholm-Gotland, SE-118 27 Stockholm, Sweden Correspondence: Jiayao Lei (jiayao.lei@ki.se)
} 


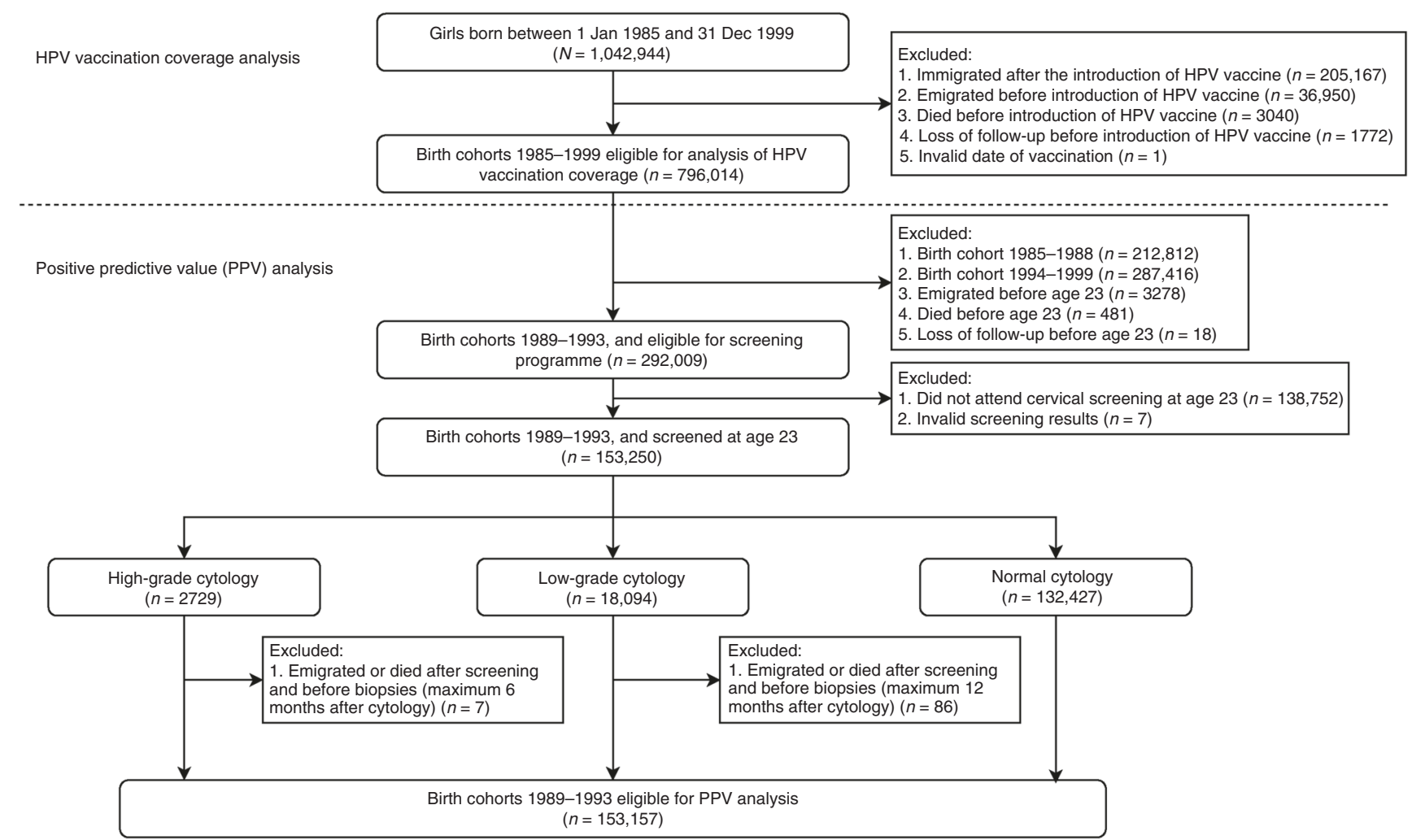

Fig. 1 Study population.

(NKCx). ${ }^{14}$ As invitations to the organised cervical screening programme in Sweden start at age 23 years, women were categorised based on their first cytology result at age 23 years as having high-grade abnormal cytology, low-grade abnormal cytology or normal cytology. Women who attended cervical screening at age 23 years and who had an abnormal cytology were followed up for histopathological assessment. We excluded women who died or who emigrated after abnormal cytology and within the required interval for histopathological assessment (6 months for high-grade cytology and 12 months for low-grade cytology, according to national guidelines at the time).

Data collection

Data were collected from the Swedish national population-based registers. All eligible women were linked through the Swedish personal identification number. ${ }^{15}$

The main exposure was defined as HPV vaccination based on a linkage to HPV vaccination records through 31 December 2017, and women with at least one dose of HPV vaccine before age 23 years were considered as vaccinated, otherwise as unvaccinated. Age at vaccination initiation was calculated, and categorised as age $<17$ and $17-22$ years based on our previous work on vaccine effectiveness against HPV-related outcomes. ${ }^{4,5,16}$ HPV vaccination information was extracted from the Swedish HPV Vaccination Register, an informed consent-based register in operation since the introduction of HPV vaccination, and complemented with data from the Prescribed Drug Register (PDR) ${ }_{1}^{17}$ which is a mandatory register on dispensed prescriptions, in operation since July 2005. Both bivalent (1.3\%) and quadrivalent (98.7\%) HPV vaccine prescriptions were identified using Anatomical Therapeutic Chemical codes.

The outcome was high-grade cervical lesions $(\mathrm{CIN} 2+)$ based on histopathological diagnoses from the NKCx, which contains all cytological and histopathological records since 1995 for all women in Sweden, from both the organised screening programme and opportunistic screening. ${ }^{14}$ Systematised Nomenclature of Medicine codes were used to define the cytological and histopathological diagnoses (Supplementary Table S1). Abnormal cytology was defined as cytological diagnoses of atypical squamous cells of undetermined significance (ASCUS) or worse. Abnormal cytology was further classified as high grade and low grade based on the severity of the cytological diagnoses. Low grade included the cytological diagnoses of ASCUS and low-grade squamous intraepithelial lesion, while the rest of the abnormal cytological diagnoses were classified as high grade, including atypical glandular cells, atypia in cells of unclear origin and CIN2 +/adenocarcinoma in situ or worse.

\section{Statistical analysis}

We estimated HPV vaccination coverage for birth cohorts 1985-1999 in total as well as by age at HPV vaccination initiation. The detection rate of high-grade cervical lesions was estimated as the proportion of women with an abnormal cytology, followed by a histopathologically confirmed CIN2+ among all women who attended screening. The PPV of cytological screening for highgrade cervical lesions was estimated as the proportion of women with an abnormal cytology and histopathologically confirmed CIN2+ among all women who had an abnormal cytology at screening. Both detection rates and PPVs were estimated separately for women with low- and high-grade cytology, considering the different clinical management strategies. All proportions were reported with $95 \%$ binomial confidence intervals (Cls). We used the log-binominal regression to estimate the ratio of PPV for $\mathrm{CIN} 2+$ in vaccinated women compared to unvaccinated women as risk ratio (RR), both crude and adjusted for birth cohort. Percentage change of PPV in vaccinated women was calculated as $(1-\mathrm{RR}) \times 100 \%$.

We performed sensitivity analyses to assess the validity of our material and the robustness of our results. First, we examined the reduction of $\mathrm{CIN} 2+$ among vaccinated women compared to 
unvaccinated women, as a comparison with other vaccinated cohorts. We also calculated the PPV for $\mathrm{CIN} 2+$ based on the first cytology test taken at any age up to 23 years, including opportunistic tests prior to screening age, to quantify the impact of opportunistic screening. In order to assess the effect of the incomplete follow-up of histopathological diagnoses for the 1993 birth cohort, we artificially applied such a truncation for all birth cohorts. Furthermore, we examined the role of referral to histopathological assessment after abnormal cytology using a histopathological diagnosis on record as a proxy for attendance at follow-up and tabulating this with vaccination status and type of abnormal cytology. Subsequently, we re-estimated the PPV of CIN2+ limited to women who had both an abnormal cytology and a follow-up histopathological diagnosis on record.

All statistical tests were two sided. SAS 9.4 was used for data management and statistical analysis. This study was approved by the Regional Ethical Review Board in Stockholm, Sweden, which determined that written informed consent by the study participants was not required.

\section{RESULTS}

Study population and vaccine coverage

We included 796,014 women born between 1985 and 1999, resident in Sweden since the introduction of HPV vaccination (1 October 2006) and age 23 years (Fig. 1). The total HPV vaccination coverage was below $10 \%$ for birth cohorts vaccinated through self-paid opportunistic vaccination (1985-1988). In birth cohorts eligible for subsidised opportunistic vaccination (1989-1992), the total vaccine coverage increased substantially and the coverage was $~ 55 \%$ among birth cohorts vaccinated through the free-of-charge, organised catch-up programme (1993-1998). The proportion of women initiating vaccination before age 17 years increased steadily by birth cohort and reached 69\% in the 1999 birth cohort (Fig. 2).

Among birth cohorts 1989-1993, a total of 153,250 women attended screening at age 23 years with valid screening results, including 2729 (1.8\%) women with high-grade cytology, 18,094 (11.8\%) women with low-grade cytology and 132,427 (86.4\%) women with a normal cytological result (Fig. 1).
Detection rate of $\mathrm{CIN} 2+$

The overall detection rate of histopathologically confirmed CIN2+ after abnormal cytology was 3.8\%, 2.3\% and $1.5 \%$ for women unvaccinated, initiating vaccination at age 17-22 years and initiating vaccination before age 17 years. Stratifying by cytological results, the detection rate of $\mathrm{CIN} 2+$ after a high-grade cytology was $1.5 \%(95 \% \mathrm{Cl}, 1.4-1.5), 0.9 \%(95 \% \mathrm{Cl}, 0.8-1.0)$ and $0.5 \%(95 \% \mathrm{Cl}, 0.5-0.6)$ for women unvaccinated, initiating vaccination at age 17-22 years and initiating vaccination before age 17 years. The complementary detection rate for $\mathrm{CIN} 2+$ after low-grade cytology were $2.3 \%(95 \% \mathrm{Cl}, 2.2-2.4), 1.4 \%(95 \% \mathrm{Cl}$, $1.3-1.5)$ and $1.0 \%(95 \% \mathrm{Cl}, 0.9-1.0)$, respectively (Table 1$)$.

PPV of cytology for CIN2+

The PPV of high-grade cytology for CIN2+ was 69.9\% $(95 \% \mathrm{Cl}$, $67.9-71.9), 64.9 \%(95 \% \mathrm{Cl}, 59.8-69.8)$ and $57.4 \%(95 \% \mathrm{Cl}$, 50.9-63.7) for women unvaccinated, initiating vaccination at age 17-22 years and initiating vaccination before age 17 years, respectively. After adjustment for birth cohorts, this corresponds to a reduction of PPV by $8 \%$ (RR $0.92,95 \% \mathrm{Cl}, 0.85-1.00)$ and $17 \%$ (RR $0.83,95 \% \mathrm{Cl}, 0.74-0.93$ ) among women initiating vaccination at age 17-22 years and women initiating vaccination before age 17 years, compared to unvaccinated women.

The PPVs of low-grade cytology for CIN2+ were $18.9 \%(95 \% \mathrm{Cl}$, 18.2-19.6), $12.8 \%$ (95\% Cl, 11.6-14.1), and 9.3\% (95\% Cl, 8.2-10.4) for women unvaccinated, women initiating vaccination at age 17-22 years and women initiating vaccination before age 17 years. Decline of PPV was observed among women initiating vaccination at age $17-22$ years with $28 \%$ (RR $0.72,95 \% \mathrm{Cl}, 0.65-0.80$ ), and women initiating vaccination before age 17 years with $44 \%$ (RR $0.56,95 \% \mathrm{Cl}, 0.49-0.63)$ compared to unvaccinated women, after adjustment for birth cohorts.

\section{Sensitivity analyses}

In the sensitivity analysis, we found 39\% and 58\% reduction of CIN2+ among women initiating vaccination at age 17-22 years and initiating vaccination before age 17 years, respectively, compared to unvaccinated women after adjustment for birth cohort (Supplementary Table S2). Calculating the PPV for CIN2+

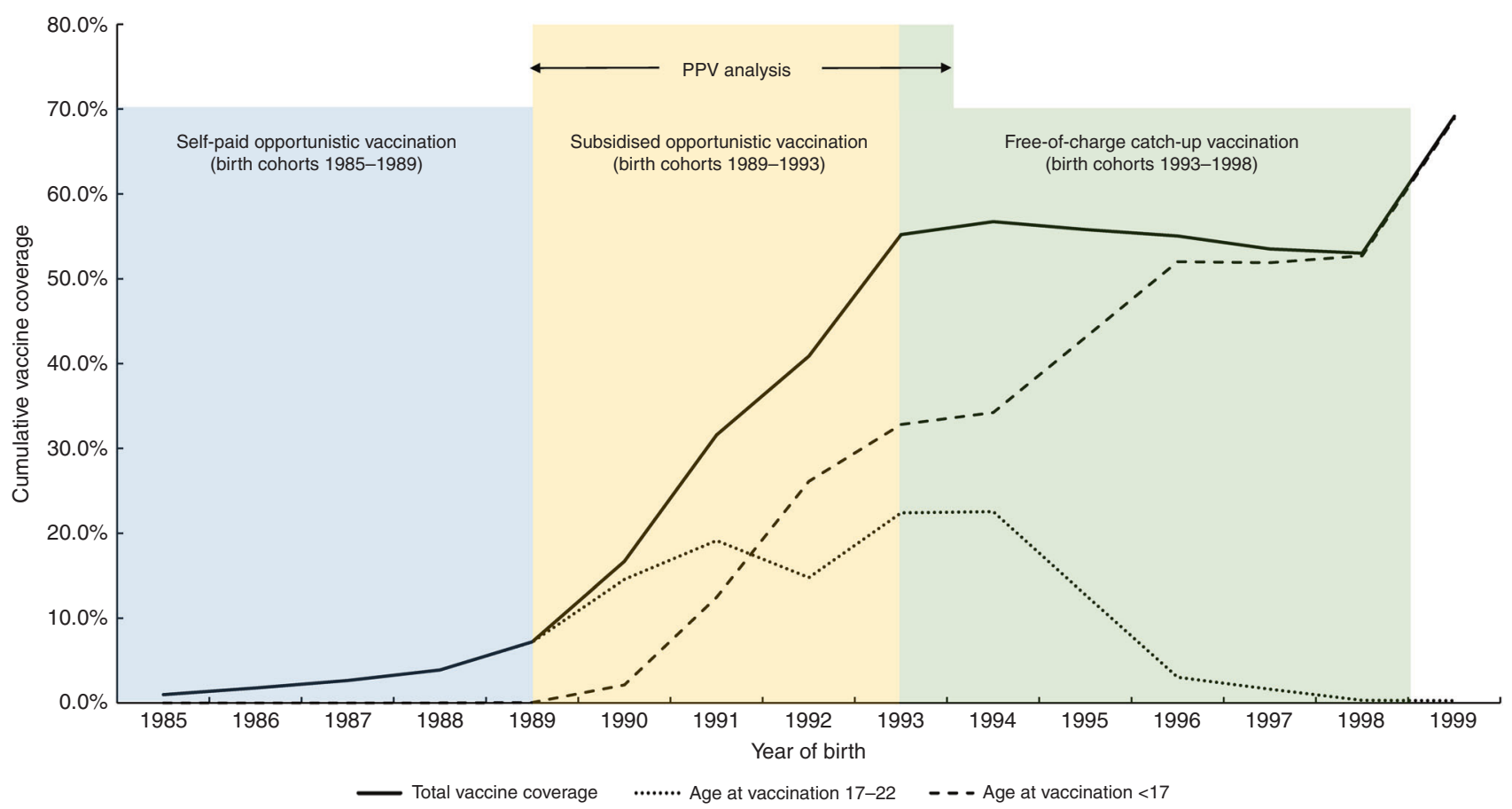

Fig. 2 Vaccination coverage for birth cohort 1985-1999, stratified by age at vaccination initiation. PPV, positive predictive value. 
J Lei et al.

Table 1. Detection rate, PPV of cytology and RRs for $\mathrm{CIN} 2+$, in relation to age at vaccination initiation.

\begin{tabular}{|c|c|c|c|c|c|c|c|c|}
\hline \multirow{2}{*}{$\begin{array}{l}\text { Cytological } \\
\text { results }\end{array}$} & \multirow{2}{*}{$\begin{array}{l}\text { Age at vaccination } \\
\text { initiation }\end{array}$} & \multirow{2}{*}{$\begin{array}{l}\text { Screened } \\
n\end{array}$} & \multirow{2}{*}{$\begin{array}{l}\text { Screen } \\
\text { positive } \\
n\end{array}$} & \multirow{2}{*}{$\begin{array}{l}\mathrm{CIN} 2+ \\
n\end{array}$} & \multirow{2}{*}{$\begin{array}{l}\text { Detection rate of } \\
\text { CIN2+, } \%(95 \% \mathrm{Cl})^{\mathrm{a}}\end{array}$} & \multicolumn{3}{|l|}{ PPV } \\
\hline & & & & & & $\begin{array}{l}\text { PPV for CIN2+, \% } \\
(95 \% \mathrm{Cl})^{\mathrm{b}}\end{array}$ & $\begin{array}{l}\text { Crude RR } \\
(95 \% \mathrm{Cl})\end{array}$ & $\begin{array}{l}\text { Adjusted }^{\mathrm{c}} \mathrm{RR} \\
(95 \% \mathrm{Cl})\end{array}$ \\
\hline \multicolumn{9}{|c|}{ High-grade cytology } \\
\hline & Unvaccinated & 100,400 & 2110 & 1475 & $1.5(1.4-1.5)$ & $69.9(67.9-71.9)$ & Reference & Reference \\
\hline & $\begin{array}{l}\text { Vaccinated at age } \\
17-22 \text { years }\end{array}$ & 26,892 & 368 & 239 & $0.9(0.8-1.0)$ & $64.9(59.8-69.8)$ & $0.93(0.86-1.01)$ & $0.92(0.85-1.00)$ \\
\hline & $\begin{array}{l}\text { Vaccinated at age } \\
<17 \text { years }\end{array}$ & 25,865 & 244 & 140 & $0.5(0.5-0.6)$ & $57.4(50.9-63.7)$ & $0.82(0.73-0.92)$ & $0.83(0.74-0.93)$ \\
\hline \multicolumn{9}{|c|}{ Low-grade cytology } \\
\hline & Unvaccinated & 100,400 & 12,293 & 2325 & $2.3(2.2-2.4)$ & $18.9(18.2-19.6)$ & Reference & Reference \\
\hline & $\begin{array}{l}\text { Vaccinated at age } \\
17-22 \text { years }\end{array}$ & 26,892 & 2940 & 377 & $1.4(1.3-1.5)$ & $12.8(11.6-14.1)$ & $0.68(0.61-0.75)$ & $0.72(0.65-0.80)$ \\
\hline & $\begin{array}{l}\text { Vaccinated at age } \\
<17 \text { years }\end{array}$ & 25,865 & 2775 & 258 & $1.0(0.9-1.0)$ & $9.3(8.2-10.4)$ & $0.49(0.44-0.56)$ & $0.56(0.49-0.63)$ \\
\hline
\end{tabular}

based on the first cytology test up to age $\leq 23$ years gave the same results as the main analysis based on the first organised screening test at age 23 years (Supplementary Table S3). After applying a data truncation for all birth cohorts, we found the decline of PPV to be robust (Supplementary Table S4). Around $90 \%$ of women with high-grade cytology in our study population had a histopathological assessment within the specified 6-month time period (Supplementary Table S5). Restricting analysis to only women with both abnormal cytology and histopathological diagnosis yielded PPV estimates comparable to our main estimates (Supplementary Table S6).

\section{DISCUSSION}

Main findings and interpretations

In this large, population-based study, we found the PPV of cytology for CIN2+ after both high- and low-grade cytology decreased significantly for vaccinated compared to unvaccinated women. The reduction of PPV was observed among birth cohorts vaccinated through either subsidised opportunistic programme or the free-of-charge catch-up programme. A stronger decrease of PPV was seen for women initiating vaccination at younger ages. The decreases of PPV corresponds to the reduced detection rates of $\mathrm{CIN2}+$ in HPV-vaccinated compared to unvaccinated women, and similarly the reduction was stronger for women initiating vaccination earlier.

Franco et al. ${ }^{12,18}$ predicted that declines in HPV infections after HPV vaccination and subsequent reduced prevalence of cervical lesions in population would inevitably lead to a decline in the PPV of cytology among HPV-vaccinated birth cohorts. We confirm the prediction $^{12,18}$ using real-life data that the decline of the PPV is related to the strength of protection that vaccination confers. Our findings also highlighted the PPV of cytology in HPV-vaccinated birth cohorts does not only depend on vaccination coverage but also on the proportion of individuals vaccinated at younger ages.

Detection rate of $\mathrm{CIN} 2+$

Lower detection rate of $\mathrm{CIN} 2+$ was observed in vaccinated women, with a more pronounced reduction among girls who initiate vaccination early (before age 17 years) compared to unvaccinated women. The reduction of $\mathrm{CIN} 2+$ among vaccinated women in our study were within the range of effectiveness of HPV vaccine against CIN2+ in other countries or regions based on an earlier systematic review. ${ }^{19}$ Moreover, a latest meta-analysis performed by Drolet et al. ${ }^{7}$ showed that HPV vaccination can effectively reduce CIN2+ by $51 \%$ and $31 \%$ in screened girls aged 15-19 and 20-24 years in post-vaccination era, respectively.

PPV of cytology for CIN2+

We found a more pronounced decline of PPV in vaccinated women after low-grade cytology compared to high-grade cytology. This could be explained by the fact that since 2015, women with low-grade cytology have been followed up with HPVreflex testing rather than direct referral to histopathological assessment. $^{20}$ This policy change has affected from birth cohort 1992 onwards, resulting in a smaller proportion of women with low-grade cytology being referred to histopathological assessment, and therefore, a lower PPV for CIN2+ after low-grade cytology.

Compared to other studies, a population-based Scottish study showed significantly lower (16\%) PPV of high-grade dyskaryosis for $\mathrm{CIN2}+$ in HPV-vaccinated women compared to unvaccinated women, based on birth cohorts vaccinated through the free-ofcharge catch-up programme. ${ }^{21}$ Another Scottish study found a lower but not statistically significant decreased PPV of colposcopy for CIN2+ in vaccinated $(66.7 \%)$ compared to unvaccinated women (74\%) in a hospital-based study. ${ }^{22}$ In an ecological study from Australia, a decreasing trend in PPV of cervical cytology was shown in both women aged $<20$ and $20-24$ years after the implementation of the HPV vaccination programme. ${ }^{23}$ Besides, a simulation study based on Danish data also suggested that the PPV of liquid-based cytology for CIN2+ declined after HPV vaccination among samples positive for any abnormalities in screening. $^{24}$

As overall vaccination coverage in Sweden has been stable, and the proportion of women initiating vaccination before age 17 years has increased steadily from birth cohort 1993 onwards (Fig. 2), we expect the PPV of cytology for CIN2+ to continue to decrease towards the level of PPV for women initiating vaccination before age 17 years. With a high coverage of HPV vaccination through the school-based programme (vaccine coverage of over $80 \%)$ and vaccination at younger ages (10-12 years) ${ }^{25}$ both direct 
protection from HPV vaccines and indirect protection gained from herd effects ${ }^{26}$ will result in very low prevalence of cervical lesions in the population. The PPV of cytology will undoubtedly reach lower levels when those birth cohorts enter the organised cervical screening in year 2022.

According to the modelling results from Franco et al., ${ }^{12,18}$ when the prevalence of cervical abnormality goes below $1 \%$, the PPV falls under $10 \%$ for the assumed levels of sensitivity. Additionally, a lower prevalence of abnormalities in cytology is likely to affect how cytologists read slides from largely vaccinated populations. ${ }^{12}$ This might potentially require change of screening methods in vaccinated cohorts, such as HPV screening. Up to now, evidence on comparing screening performance of cytology screening and HPV screening in HPV-vaccinated cohorts is sparse. In a follow-up study of the Compass pilot randomised trial, ${ }^{27}$ an increased detection of $\mathrm{CIN} 2+$ in highly HPV-vaccinated birth cohorts by using HPV primary screening compared to using cytology was observed, and the referral rate for colposcopy was not higher.

Limitations and strengths of this study

We did not have complete follow-up of histopathological diagnoses for the 1993 birth cohort due to right truncation of our data. Second, non-attendance to histopathological assessment following an abnormal cytology could potentially influence the PPV. However, overall $96 \%$ of women with high-grade cytology have a histopathological assessment within 1 year, ${ }^{14}$ and we also observed a high attendance to histopathological assessment in our study population. Furthermore, results from sensitivity analyses supported the robustness of our estimates even when accounting for the above-mentioned limitations (Supplementary Tables S2-S9). Finally, a small proportion of HPV-vaccinated women in birth cohort 1993 (corresponding to $6.2 \%$ of vaccination doses in that birth cohort) could be misclassified as unvaccinated due to incomplete vaccination registration, even if the incompleteness of HPV registration was to a large extent supplemented by further linkages to the PDR. However, the misclassifications will result in an underestimation of the detection rate of CIN2+ and the PPV for unvaccinated women, with limited impact on estimates for vaccinated women, meaning that the results will be attenuated.

Our study investigated the PPV of cytology for CIN2+ based on the population-based, organised screening programme with individual linkage to HPV vaccination status in a nationwide setting. As the PPV is strongly influenced by the management of low-grade lesions, our strategy to evaluate the PPV by low- and high-grade cytology separately limits the bias in PPV comparisons. Allowing a different timeframe for women with low- and highgrade cytology to attend histopathological assessment reflects the clinical management for different severity of abnormalities in cytology. Moreover, cytological tests taken before age 23 years might represent a different risk profile, so only considering the first cytology taken at age 23 years as outcome could minimise possible selection bias. Individual level linkages to high-quality Swedish registries ensured identification of exposure and outcome measures, while minimising misclassification. Besides, the cervical screening data routinely collected through NKCx provides complete coverage of cytological and histopathological diagnoses from both organised screening and opportunistic screening. ${ }^{14}$ Finally, reporting percentage changes of PPV enhances the generalisability of our findings. It offers other countries or regions with comparable HPV vaccination and cervical screening programmes to estimate their expected level of PPV among HPVvaccinated birth cohorts based on current level of PPV, vaccination coverage and age at vaccination initiation.

Public health relevance and future research

Our study provides direct evidence that the PPV of abnormal cytology for CIN2+ declines with HPV vaccination, particularly when initiated at young ages. The findings should be generalisable to other countries or regions with effective HPV vaccination and organised cervical screening programmes. As additional birth cohorts with higher HPV vaccination coverage enter the screening programmes in the coming years, the PPV is likely to decline further.

The change of PPV for CIN2+ after low-grade cytology also highlights the importance of pragmatically adapting the screening programme to an increasing proportion of low-grade cytology, with low cancer risk. A reduced prevalence of HPV16/18 is confirmed by an earlier study, indicating that most of the cervical intraepithelial neoplasia among vaccinated cohorts were associated with non-vaccine HPV types. $^{28}$ Therefore, HPV16/18 positivity in vaccinated birth cohorts entering the screening programme will most likely be an indication of persistent infections, for example, if vaccination was initiated after prior exposure to HPV. This suggests that either decreased intensity and/or switch to primary HPV screening with partial HPV genotyping should be contemplated.

In the future, surveillance of HPV infections among HPVvaccinated cohorts and additional comparative effectiveness studies on the PPV of cytology and HPV testing for high-grade cervical lesions are needed. This could further demonstrate the optimal screening method for HPV-vaccinated birth cohorts with corresponding age of starting screening and screening intervals. Additionally, a re-evaluation of screening performance when birth cohorts vaccinated with 9-valent vaccine enter the screening programme would also be warranted.

\section{CONCLUSION}

HPV-vaccinated women had a decreased PPV of cytology for CIN2+ compared to unvaccinated women. The PPV of cytology for CIN2+ also showed a stronger decrease among women initiating vaccination before age 17 years compared to women initiating vaccination at age 17-22 years relative to unvaccinated women. The performance of cytology-based screening is expected to further decline in cohorts with higher vaccination coverage, potentially impacting the choice of optimal screening strategies for younger women. Primary HPV screening with partial genotyping could be an alternative screening method for HPV-vaccinated cohorts.

\section{ACKNOWLEDGEMENTS}

We thank all study participants contributing data to our research. We acknowledge Pouran Almstedt for data management.

\section{AUTHOR CONTRIBUTIONS}

J.L. did the literature search and review, analysed the data, and wrote the original draft of this manuscript. J.D., K.M.E., M.L. and P.S. conceived the research questions and hypotheses. J.D., P.S., M.L. and K.M.E. designed the study. J.D. and P.S. collected and standardised the cervical screening data. A.P. advised on statistical analysis. J.L., A.P., K.M.E., J.D., M.L. and P.S. interpreted the data. All authors are involved in the critical revision of article and have read and approved submission of the manuscript.

\section{ADDITIONAL INFORMATION}

Ethics approval and consent to participate This study was approved by the Regional Ethical Review Board in Stockholm, Sweden (Dnr. 02-556; Dnr. 2011/921-32; Dnr. 2012/216-32 and Dnr. 2012/1426-32), which determined that written informed consent by the study participants was not required. The study was performed in accordance with the Declaration of Helsinki.

Data availability Data from the study is available on request from corresponding author.

Competing interests J.D. is formerly recipient of grants on HPV vaccine research from Merck/SPMSD, and grants to his employer (Karolinska University Hospital) from 
Roche and Genomica; M.L. has received grants for his HPV vaccination studies from Merck \& Co., Inc. and GSK Biologicals through his employers T.H.L. and Tampere University; the other authors have no conflict of interest to declare; no financial relationships with any organisations that might have an interest in the submitted work in the previous three years; no other relationships or activities that could appear to have influenced the submitted work.

Funding information This work was supported by CoheaHr (FP7-F3-2013-603019), Swedish Foundation for Strategic Research (KF10-0046); Swedish Cancer Society (CAN 2016/840); Swedish Research Council (2017-02346) and the China Scholarship Council (201507930001)

Role of sponsor The study sponsor did not participate in study design, data collection, analysis, interpretation of data, writing of the article or the decision to submit it for publication.

Supplementary information is available for this paper at https://doi.org/10.1038/ s41416-020-0850-6.

Publisher's note Springer Nature remains neutral with regard to jurisdictional claims in published maps and institutional affiliations.

\section{REFERENCES}

1. Walboomers, J. M., Jacobs, M. V., Manos, M. M., Bosch, F. X., Kummer, J. A., Shah, K. $V$. et al. Human papillomavirus is a necessary cause of invasive cervical cancer worldwide. J. Pathol. 189, 12-19 (1999).

2. Immunization, W. Vaccines and Biologicals Database. http://www.who.int/entity/ immunization/monitoring_surveillance/VaccinelntroStatus.pptx (2019). Accessed 5 December 2019.

3. Wang, J., Ploner, A., Sparen, P., Lepp, T., Roth, A., Arnheim-Dahlstrom, L. et al. Mode of HPV vaccination delivery and equity in vaccine uptake: a nationwide cohort study. Prev. Med. 120, 26-33 (2019).

4. Herweijer, E., Sundström, K., Ploner, A., Uhnoo, I., Sparén, P. \& Arnheim-Dahlström, L. Quadrivalent HPV vaccine effectiveness against high-grade cervical lesions by age at vaccination: a population-based study. Int. J. Cancer 138, 2867-2874 (2016).

5. Herweijer, E., Sundström, K., Ploner, A., Uhnoo, I., Sparén, P. \& Arnheim-Dahlström, L. Erratum. Int. J. Cancer 141, E1-E4 (2017).

6. Herweijer, E., Ploner, A. \& Sparen, P. Substantially reduced incidence of genital warts in women and men six years after HPV vaccine availability in Sweden. Vaccine 36, 1917-1920 (2018).

7. Drolet, M., Benard, E., Perez, N. \& Brisson, M., Group HPVVIS. Population-level impact and herd effects following the introduction of human papillomavirus vaccination programmes: updated systematic review and meta-analysis. Lancet https://doi.org/10.1016/S0140-6736(19)30298-3 (2019).

8. Malagon, T., Drolet, M., Boily, M. C., Franco, E. L., Jit, M., Brisson, J. et al. Crossprotective efficacy of two human papillomavirus vaccines: a systematic review and meta-analysis. Lancet Infect. Dis. 12, 781-789 (2012).

9. Bonanni, P., Boccalini, S. \& Bechini, A. Efficacy, duration of immunity and cross protection after HPV vaccination: a review of the evidence. Vaccine 27(Suppl. 1), A46-A53 (2009).

10. Lehtinen, M. \& Dillner, J. Clinical trials of human papillomavirus vaccines and beyond. Nat. Rev. Clin. Oncol. 10, 400-410 (2013).

11. von Karsa, L., Arbyn, M., De Vuyst, H., Dillner, J., Dillner, L., Franceschi, S. et al. European guidelines for quality assurance in cervical cancer screening. Summary of the supplements on HPV screening and vaccination. Papillomavirus Res. 1, 22-31 (2015).

12. Franco, E. L., Mahmud, S. M., Tota, J., Ferenczy, A. \& Coutlee, F. The expected impact of HPV vaccination on the accuracy of cervical cancer screening: the need for a paradigm change. Arch. Med. Res. 40, 478-485 (2009).

13. Ludvigsson, J. F., Almqvist, C., Bonamy, A. K., Ljung, R., Michaelsson, K., Neovius, $M$. et al. Registers of the Swedish total population and their use in medical research. Eur. J. Epidemiol. 31, 125-136 (2016).

14. Swedish National Cervical Screening Registry (NKCx). Prevention of Cervical Cancer in Sweden. Annual report. http://www.nkcx.se/templates/_rsrapport_2018.pdf (2018). Accessed 24 Apr 2019.
15. Ludvigsson, J. F., Otterblad-Olausson, P., Pettersson, B. U. \& Ekbom, A. The Swedish personal identity number: possibilities and pitfalls in healthcare and medical research. Eur. J. Epidemiol. 24, 659-667 (2009).

16. Leval, A., Herweijer, E., Ploner, A., Eloranta, S., Fridman Simard, J., Dillner, J. et al. Quadrivalent human papillomavirus vaccine effectiveness: a Swedish national cohort study. J. Natl Cancer Inst. 105, 469-474 (2013).

17. Wettermark, B., Hammar, N., Fored, C. M., Leimanis, A., Otterblad Olausson, P., Bergman, U. et al. The new Swedish Prescribed Drug Register-opportunities for pharmacoepidemiological research and experience from the first six months. Pharmacoepidemiol. Drug Saf. 16, 726-735 (2007).

18. Franco, E. L., Cuzick, J., Hildesheim, A. \& de Sanjose, S. Chapter 20: issues in planning cervical cancer screening in the era of HPV vaccination. Vaccine $\mathbf{2 4}$ (Suppl. 3), S3/171-S3/17177 (2006).

19. Garland, S. M., Kjaer, S. K., Munoz, N., Block, S. L., Brown, D. R., DiNubile, M. J. et al. Impact and effectiveness of the quadrivalent human papillomavirus vaccine: a systematic review of 10 years of real-world experience. Clin. Infect. Dis. 63, 519-527 (2016).

20. Regional Cancer Centers in Sweden in Cooperation. National Swedish Guidelines for Cervical Cancer Prevention, Diagnosis, and Treatment. https://www. cancercentrum.se/globalassets/vara-uppdrag/prevention-tidig-upptackt/ gynekologisk-cellprovskontroll/vardprogram/nationellt-vardprogramcervixcancerprevention.pdf (2019). Accessed 16 Jan 2020 [In Swedish].

21. Palmer, T. J., McFadden, M., Pollock, K. G., Kavanagh, K., Cuschieri, K., Cruickshank, M. et al. HPV immunisation and cervical screening-confirmation of changed performance of cytology as a screening test in immunised women: a retrospective population-based cohort study. Br. J. Cancer 114, 582-589 (2016)

22. Munro, A., Gillespie, C., Cotton, S., Busby-Earle, C., Kavanagh, K., Cuschieri, K. et al. The impact of human papillomavirus type on colposcopy performance in women offered HPV immunisation in a catch-up vaccine programme: a two-centre observational study. BJOG 124, 1394-1401 (2017).

23. Sultana, F., Winch, K., Saville, M. \& Brotherton, J. M. L. Is the positive predictive value of high-grade cytology in predicting high-grade cervical disease falling due to HPV vaccination? Int. J. Cancer https://doi.org/10.1002/ijc.32050 (2018).

24. Hestbech, M. S., Lynge, E., Kragstrup, J., Siersma, V., Vazquez-Prada Baillet, M. \& Brodersen, J. The impact of HPV vaccination on future cervical screening: a simulation study of two birth cohorts in Denmark. BMJ Open 5, e007921 (2015).

25. Statistics for HPV vaccinations-share of vaccinated girls through 2015-12-31. https://www.folkhalsomyndigheten.se/globalassets/statistik-uppfoljning/ vaccinationsstatistik/hpv/statistik-for-hpv-vaccinationer-andel-vaccineradeflickor-tom-2015-12-31.pdf (2015). Accessed 16 Aug 2017.

26. Drolet, M., Benard, E., Boily, M. C., Ali, H., Baandrup, L., Bauer, H. et al. Populationlevel impact and herd effects following human papillomavirus vaccination programmes: a systematic review and meta-analysis. Lancet Infect. Dis. 15, 565-580 (2015).

27. Canfell, K., Caruana, M., Gebski, V., Darlington-Brown, J., Heley, S., Brotherton, J. et al. Cervical screening with primary HPV testing or cytology in a population of women in which those aged 33 years or younger had previously been offered HPV vaccination: results of the Compass pilot randomised trial. PLoS Med. 14, e1002388 (2017).

28. Kann, H., Hortlund, M., Eklund, C., Dillner, J. \& Faust, H. Human papillomavirus types in cervical dysplasia among young HPV-vaccinated women: populationbased nested case-control study. Int. J. Cancer https://doi.org/10.1002/ijc.32848 (2019).

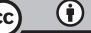

Open Access This article is licensed under a Creative Commons Attribution 4.0 International License, which permits use, sharing, adaptation, distribution and reproduction in any medium or format, as long as you give appropriate credit to the original author(s) and the source, provide a link to the Creative Commons license, and indicate if changes were made. The images or other third party material in this article are included in the article's Creative Commons license, unless indicated otherwise in a credit line to the material. If material is not included in the article's Creative Commons license and your intended use is not permitted by statutory regulation or exceeds the permitted use, you will need to obtain permission directly from the copyright holder. To view a copy of this license, visit http://creativecommons. org/licenses/by/4.0/.

(c) The Author(s) 2020 